\title{
Lower carbohydrate versus lower fat: a systematic review and meta-analysis of dietary interventions for reducing liver fat in metabolic associated fatty liver disease
}

\author{
L. Zhou ${ }^{1}$, M. Holmes ${ }^{1}$, M. Zulyniak ${ }^{1}$ and J.B. Moore ${ }^{1}$ \\ ${ }^{1}$ School of Food Science and Nutrition, University of Leeds, Leeds, UK
}

Closely associated with obesity, metabolic-dysfunction associated fatty liver disease (MAFLD) is now the most common cause of chronic liver disease and a significant public health problem worldwide ${ }^{(1)}$. Although $5-10 \%$ weight loss is typically recommended for reducing liver fat (intrahepatocellular triglyceride, IHTG) and other markers of MAFLD, this can be difficult for patients to achieve ${ }^{(2)}$. Moreover, the relative roles of dietary macronutrients in the pathogenesis of MAFLD are only partially understood and questions remain about optimal dietary interventions for MAFLD treatment. Therefore, the aim of this work was to systematically evaluate and synthesize the evidence from intervention trials investigating the efficacy of lower carbohydrate diets (LCD; $\leq 45 \%$ energy) in comparison to lower fat diets (LFD) on reducing IHTG content and body weight.

This systematic review and meta-analysis was conducted by independent reviewers in accordance with PRISMA guidelines and prospectively registered at Prospero (CRD42021223085). The MEDLINE (via PubMed), Scopus, EMBASE and Cochrane electronic databases were systematically searched from inception through December 14th 2020 for randomized or crossover intervention trials assessing the effects of LCD or LFD on IHTG (measured by MRS or MRI) and body weight. Risk of publication bias and study quality was assessed using the Cochrane Risk of Bias II (ROBINS-II) tool. The R software environment was used to conduct meta-analyses using the inverse variance method and random effects models. Heterogeneity between studies was evaluated with Cochran's Q-test and the I-squared index. Comparison between subgroups was done using a plural, fixed effects, model.

Fifteen studies, including 12 randomized intervention trials and 3 crossover studies reporting on IHTG as a primary outcome were identified and summarized in the qualitative synthesis. Of the 12 trials, 6 were isoenergetic and 6 were hypoenergetic interventions, while all 3 crossover studies were isoenergetic. Examining effect sizes as weighted mean difference (WMD) from baseline to intervention endpoint showed that both LCD and LFD significantly reduced IHTG (WMD [95\% confidence intervals]: LCD: $-2.38 \%$ [-3.81, $-0.95]$ versus LFD: $-1.83 \%$ [-2.91, -0.75$] ; \mathrm{P}=0.54$ ) and body weight (LCD: $-3.99 \mathrm{~kg}$ [-6.06, -1.92$]$ versus LFD: $-3.38 \mathrm{~kg} ;[-5.63,-1.13] ; \mathrm{P}$ $=0.70$ ), with no difference in effect size between them. However, significant differences were observed between hypocaloric and isocaloric interventions, with hypocaloric trials demonstrating a stronger effect for IHTG (HYPO: $-3.82 \%$ [-4.95, -2.69] versus ISO: $-0.81 \%[-1.52,-0.09] ; \mathrm{p}<0.00001)$ and body weight (HYPO: $-6.77 \mathrm{~kg}[-8.94,-4.6]$ versus ISO: $-0.73 \mathrm{~kg}[-2.88,-1.4] ; \mathrm{p}<0.0001$ ).

In conclusion these data show hypocaloric interventions have a stronger effect on IHTG removal and weight loss than isocaloric diets in MAFLD. Interventions decreasing either carbohydrate or fat intakes were similarly effective, suggesting macronutrient composition is less important than total energy intake and choice may be based on individual preference to promote adherence.

\section{References}

1. Moore JB (2019) Proc Nutr Soc 78, 290-304.

2. Kenneally S, Sier JH \& Moore JB (2017) BMJ Open Gastro 4, e000139. 